\title{
Internacionalização da produção científica do Brasil em física de altas energias (1983-2013)
}

Internationalization of scientific output in the field of high energy physics in Brazil (1983-2013)

\author{
Gonzalo Rubén Alvarez ${ }^{1}$, Sônia Elisa Caregnato ${ }^{1}$ \\ ${ }^{1}$ Universidade Federal do Rio Grande do Sul
}

\begin{abstract}
RESUMO
Este estudo bibliométrico analisa as dimensões da internacionalização da produção científica brasileira em Física de Altas Energias (FAE) a partir dos artigos publicados em revistas indexadas pela Web of Science (WoS) no período de 1983-2013. Com base na classificação proposta pelo Manual de Santiago da Red de Indicadores de Ciencia y Tecnologia Iberoamericana e Interamericana (RICYT), a internacionalização do Brasil em FAE é estimada através de Indicadores de Difusão, Colaboração e Impacto Internacional. Os indicadores de Difusão Internacional revelam a preferência pela publicação em periódicos estrangeiros de língua inglesa. A totalidade dos artigos analisados foi veiculada em 35 revistas de 11 países diferentes. Os indicadores de Colaboração Internacional mostram a prevalência da coautoria em FAE visto que 49,07\% dos artigos foram assinados por mais de um país. Os indicadores de Impacto Internacional destacam $o$ peso das publicações em periódicos estrangeiros de língua inglesa dentre os citantes. Os documentos citantes internacionais representam $87,78 \%$ do total, com destaque para os assinados por autores dos Estados Unidos. Os indicadores de internacionalização utilizados desvelam o perfil científico internacional da FAE no Brasil, acompanhando o padrão característico da área na esfera global.
\end{abstract}

PAlAVRAS-ChaVE: Produção científica. Internacionalização. Bibliometria. Física de Altas Energias.

\section{ABSTRACT}

This bibliometric study analyzes the dimensions of internationalization of Brazilian scientific output in the field of High Energy Physics (HEP) from articles published in journals indexed by Web of Science (WoS) in the 1983-2013 period. Based on the classification proposed by the Manual of Santiago from Red de Indicadores de Ciencia y Tecnologia Iberoamericana e Interamericana (RICYT), internationalization of Brazil in HEP is estimated by Diffusion Indicators, Collaboration and International Impact. Diffusion International indicators show a preference for publishing in foreign journals using English language. All of the analyzed articles were published in 35 journals from 11 different countries. International Collaboration indicators show the prevalence of co-authorship in HEP, as authors from more than one country signed $49.07 \%$ of the articles. International Impact indicators highlight the weight of citations coming from publications in foreign journals written in English. International citing documents represent $87.78 \%$ of the total, particularly those signed by US authors. Internationalization indicators unveil the international scientific profile of Brazilian HEP, following the pattern of the field worldwide.

KEYWORDS: Scientific output. Internationalization. Bibliometric. High Energy Physics.

\section{Correspondência}

${ }^{1}$ Gonzalo Rubén Alvarez

Universidade Federal do Rio Grande do Sul.

Porto Alegre, RS.

Email: gonzalorubenalvarez@gmail.com

ORCID: http://orcid.org/0000-0002-0677-5865.

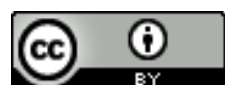

JITA: BB. Bibliometric methods. 


\section{INTRODUÇÃ̃O}

A mudança na organização da pesquisa científica, de Little Science para Big Science, aportou novos conhecimentos sobre as redes de colaboração científica. Desde o advento da 'Grande Ciência', o número de autores por artigo tem aumentado significativamente, especialmente na Física de Altas Energias (FAE), onde as investigações somente podem ser realizadas graças ao trabalho em colaboração internacional devido ao custo e complexidade dos experimentos com aceleradores de partículas (KRETSCHMER; ROUSSEAU, 2001). Na atualidade, o modelo teamwork ou colaboração gigante representa o novo paradigma e manifesta a mudança no modo de produção do conhecimento.

Em vista disso, a internacionalização é percebida como uma condição necessária para o desenvolvimento da ciência, assim como um canal para a melhora da qualidade das atividades científicas e tecnológicas, a formação de recursos humanos, a circulação de informação e o fortalecimento dos vínculos entre parceiros (RED..., 2007). Mele et al. (2006) conceituam à FAE como uma das disciplinas científicas mais internacionalizada e colaborativa. Desde a década de 1950, grandes projetos experimentais têm caráter participativo, contando com centenas de pesquisadores de vários países. As instalações científicas e tecnológicas multinacionais, como por exemplo, o laboratório European Organization for Nuclear Research (CERN), constituem uma importante modalidade de internacionalização a partir do trabalho em colaboração, especialmente em campos como a Astronomia, Física de Partículas e Física Nuclear (RED..., 2007).

A internacionalização da ciência pode ser ponderada com base em diferentes indicadores: autoria, difusão e impacto internacional. A produção científica é um elemento essencial na avaliação do conhecimento por indicadores dado que oferece informação sobre o desempenho e a contribuição de agregados para a produção mundial, a intensidade da colaboração com o exterior, a visibilidade e a influência internacional da investigação de disciplinas/países através das citações recebidas, estabelecendo bases para a elaboração de políticas para a internacionalização da ciência (RED..., 2007). Alguns autores como van Raan (1997) expressam que o processo de internacionalização de um país deve incluir a publicação em revistas internacionais de língua inglesa com alto fator de impacto e a colaboração com autores estrangeiros visto que eleva o impacto internacional. Wang, Wang e Weldon (2007) acrescentam que as citações de todas as nacionalidades são também um aspecto importante a ser considerado na melhoria dos níveis de globalização.

Em 2008, o Brasil começou as negociações no CERN para se tornar, sob uma nova modalidade de participação, um Membro Associado. Apesar de não possuir um vínculo formal com o laboratório, a colaboração da FAE brasileira é antiga e intensa. Em número de pesquisadores, alunos e engenheiros, o Brasil é o segundo país não-membro com maior participação, atrás apenas do Canadá. Igualmente, cientistas brasileiros participam dos grandes projetos internacionais envolvendo colaborações em Laboratórios Nacionais como o Fermi National Accelerator Laboratory (Fermilab) e Brookhaven National Laboratory (BNL)

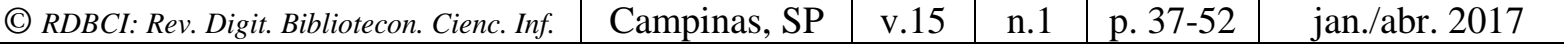


nos Estados Unidos e Pierre Auger na Argentina (COORDENAÇÃO..., 2013a), dando mostras do desempenho do Brasil em FAE. No mesmo ano, o Ministério de Ciência e Tecnologia (MCT) instituiu a Rede Nacional de Física de Altas Energias (RENAFAE), cujos objetivos principais visam coordenar as atividades relacionadas às grandes colaborações multinacionais e aumentar a intensidade da internacionalização no âmbito institucional (CENTRO..., 2011; SHELLARD, 2011).

Chaves et al. (2007) consideram que a maturidade e a qualidade alcançada pela FAE brasileira refletem no número de colaborações internacionais e publicações em coautoria. No Brasil, a área se constitui como uma das disciplinas com maior tradição na pesquisa experimental. O seu desenvolvimento fica comprovado pela representatividade dos grupos de pesquisa $\mathrm{CNPq}$ e qualidade dos programas de pós-gradução com linhas de pesquisa FAE, sendo que 11 dos 32 PPGs (8 com nota 7 e 3 com nota 6) são considerados com perfil internacional e de excelência segundo a Avaliação Trienal 2013 da CAPES.

Historicamente, a Física de Altas Energias é vinculada ao cenário internacional, tanto pela chegada de pesquisadores estrangeiros ao Brasil no início da década de 1930 quanto pela forte cultura de comunicação dos resultados das pesquisas e intercâmbio científico entre laboratórios de diferentes países. Estudos anteriores destacam a magnitude da colaboração no que se refere ao número de países presentes nos artigos e à preferência dos cientistas da FAE mundial quanto à publicação das descobertas em prestigiosas revistas internacionais de alto Fator de Impacto (FI) para a Física de Partículas (BRAUN et al., 1992; MELE et al., 2006; KRAUSE; LINDQVIST; MELE, 2007; AMAN, 2013). Conforme Duarte (2008), a intensificação das relações com os Estados-Membros do CERN em termos de Cooperação Internacional pode representar uma janela de oportunidades para o Brasil nos próximos anos visto que a troca de experiências científicas envolvendo dois ou mais países agrega conhecimento para a publicação de artigos com a participação de múltiplos autores e propicia o intercâmbio de experiências, métodos e processos nos grandes empreendimentos e estruturas multinacionais. Portanto, a importância deste estudo reside na possibilidade de expandir os conhecimentos sobre a internacionalização da FAE brasileira e fornecer subsídios para o planejamento de políticas e estratégias atinentes à gestão para o ingresso do Brasil no CERN sob uma nova modalidade de participação.

Esta pesquisa bibliométrica analisa as dimensões da internacionalização da produção científica brasileira em FAE a partir dos artigos publicados em revistas indexadas pela Web of Science (WoS) no período de 1983-2013. Com base na classificação proposta pelo Manual de Santiago da Red de Indicadores de Ciencia y Tecnología Iberoamericana e Interamericana (RICYT), a internacionalização da FAE brasileira foi estimada através de Indicadores de Difusão, Colaboração e Impacto Internacional. 


\section{METODOLOGIA}

O corpus de análise deste estudo bibliométrico é composto pelos artigos da FAE brasileira indexados na base multidisciplinar WoS no período de 1983-2013. A coleta e download dos registros foi realizada em dezembro de 2014, utilizando a opção de Busca Avançada. Na estratégia de busca foram utilizados os rótulos de campo CU=País (Brazil OR Brasil) e WC=Categoria Web of Science (Physics, Particles \& Fields). Os resultados foram limitados ao tipo de documento Article, ao idioma All languages, ao tempo estipulado de 1983 até 2013, ao índice de citações Science Citation Index (SCI). Após a importação dos 6.350 artigos a serem utilizados para as análises de difusão e colaboração internacional, foi efetuada a organização das informaçãoes em um arquivo único (.txt). Na padronização dos nomes das instituições e países colaboradores/citantes foram utilizadas a Lista de Autoridades do Grupo de Pesquisa Comunicação Científica da Universidade Federal do Rio Grande do Sul (UFRGS) e a Plataforma Lattes do CNPq. Para análise de impacto internacional, os dados dos 41.152 documentos citantes foram importados a partir da opção Criar Relatório de Citações (Create Citation Report) da WoS.

As variáveis analisadas foram Ano de Publicação (PY), Periódico (SO), Idioma (LA), Endereço de Autor (C1). Com base na classificação proposta pela RICYT e em concordância com a metodologia adotada por Santin, Vanz e Stumpf (2015), na análise da internacionalização da produção científica da FAE brasileira foram utilizados Indicadores de Difusão Internacional (publicação em periódicos estrangeiros de língua inglesa), Colaboração Internacional (coautoria com autores de outros países), Impacto Internacional (citações em publicações de autores internacionais). Com relação ao tratamento dos dados, foram empregados os software Bibexcel (análises descritivas), Pajek (rede de colaboração), Philcarto (mapeamento temático) e Microsoft Excel 2007. Na geração dos indicadores de colaboração e impacto internacional, este estudo utilizou a contagem total, ou seja, um artigo para cada país colaborador/citante.

\section{RESULTADOS}

O conjunto de artigos brasileiros representa $3 \%$ do total da produção científica mundial da FAE indexada na WoS no período de 1983-2013, com média anual de crescimento de 14,27\%. Na Figura 1 é possível observar o desenvolvimento da atividade em FAE ao longo da série temporal. A indexação de novos periódicos brasileiros por parte da WoS no biênio 2007-2008 e o consequente aumento verificado na produção científica do Brasil não afetou à area visto que foi observada uma taxa de crescimento negativa em 2008 ($18,91 \%)$. Os indicadores de difusão internacional apresentados a continuação confirmam a independência da FAE quanto aos veículos nacionais de comunicação. 


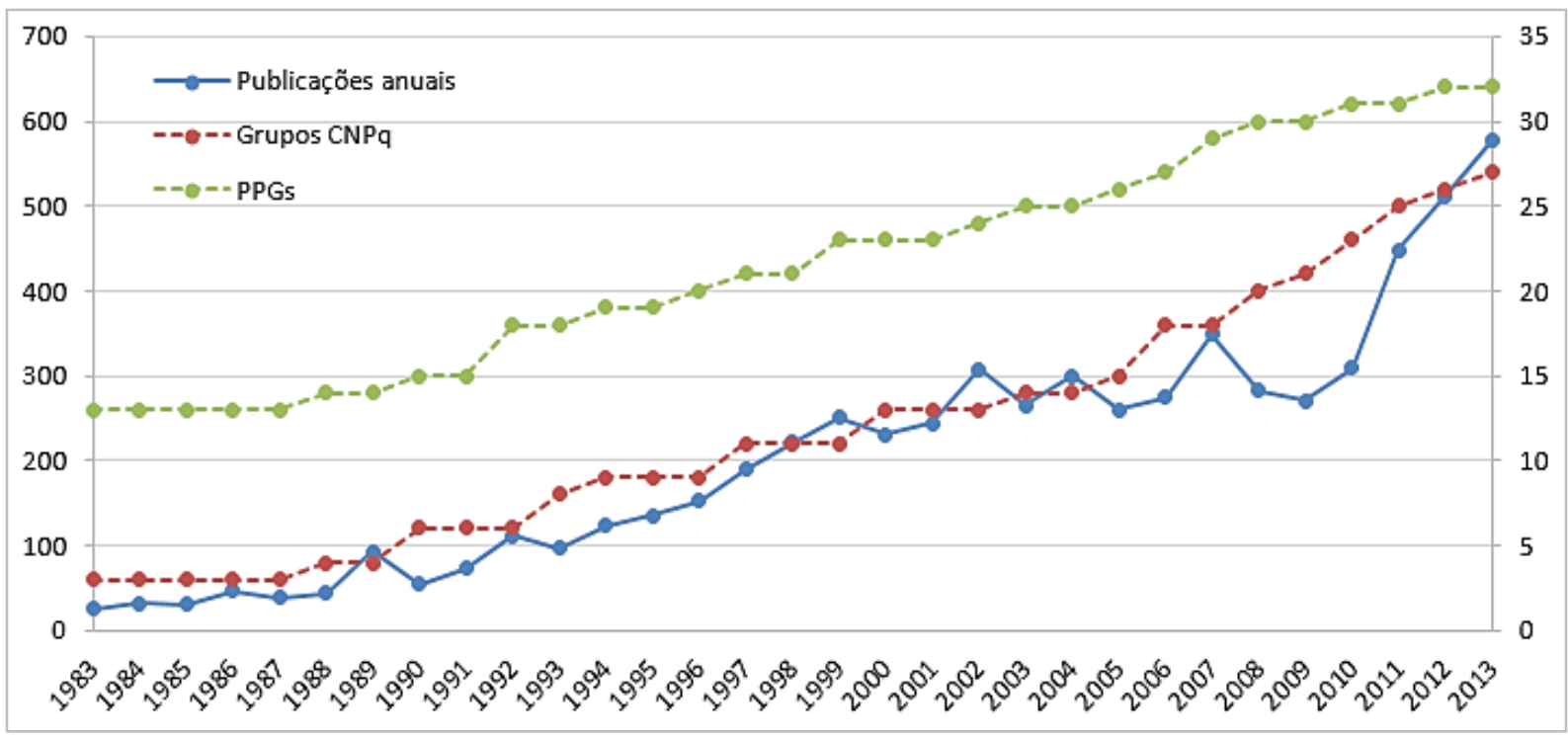

Figura 1. Dinâmica de crescimento das publicações anuais, Grupos CNPq e PPGs da FAE brasileira (1983-2013)

Fonte: dados da pesquisa

$\mathrm{O}$ aumento da produtividade da FAE pode ter sido decorrência da expansão da pesquisa a partir da criação de novos programas de pós-graduação e grupos CNPq (Figura 1). Percebe-se que o papel articulador da RENAFAE foi fundamental para a área visto que o número de publicações e grupos $\mathrm{CNPq}$ aumentou consideravelmente a partir de 2008. A ampliação dos PPGs e instituições de pesquisa das regiões Sul, Nordeste e Norte na década de 2000 pode também ter promovido o incremento da produção do Brasil em FAE.

\subsection{Difusão Internacional}

A difusão internacional da FAE brasileira foi analisada em relação ao percentual de artigos em periódicos estrangeiros de língua inglesa e à distribuição geográfica da produção científica indexada na WoS conforme país editor das publicações. Os 6.350 artigos brasileiros foram veiculados em 35 periódicos de 11 países diferentes. O conjunto de publicações está concentrado em revistas internacionais especializadas de língua inglesa (três multilíngues). Os títulos editados na Europa (Alemanha, Inglaterra, Holanda, Itália, Rússia, Suíça, Ucrânia) com 2.963 artigos $(46,67 \%)$ foram os mais produtivos. A seguir, surgem os periódicos norteamericanos com $2.515(39,60 \%)$ e os asiáticos (Singapura, China, Tailândia) com 872 $(13,73 \%)$ publicações. A distribuição geográfica da pesquisa do Brasil em FAE denota a concentração das publicações em um número reduzido de países editores (Figura 2). Estados Unidos com 10 títulos $(28,58 \%)$ é o país com maior número de periódicos editados. Em seguida, aparecem Inglaterra com 5 (14,29\%); Alemanha e Holanda com 4 cada um (11,42\%); Singapura com 3 (8,58\%); Itália, Rússia e China com 2 cada um (5,71\%); Suíça, Ucrânia e Tailândia com 1 cada um (2,86\%). 
Leite, Mugnaini e Leta (2011) constataram que quanto mais experimental e/ou tecnológica é a disciplina, maior é a fração de cientistas com perfil altamente internacional. $\mathrm{Na}$ análise, as Ciências Exatas e da Terra (incluída a FAE), a Biologia e a Engenharia foram os campos que apresentaram os índices mais elevados. Os autores entendem que para alcançar um perfil altamente internacional, as áreas precisam publicar entre $80 \%$ e $100 \%$ dos trabalhos em periódicos estrangeiros de língua inglesa, comportamento observado na FAE brasileira.

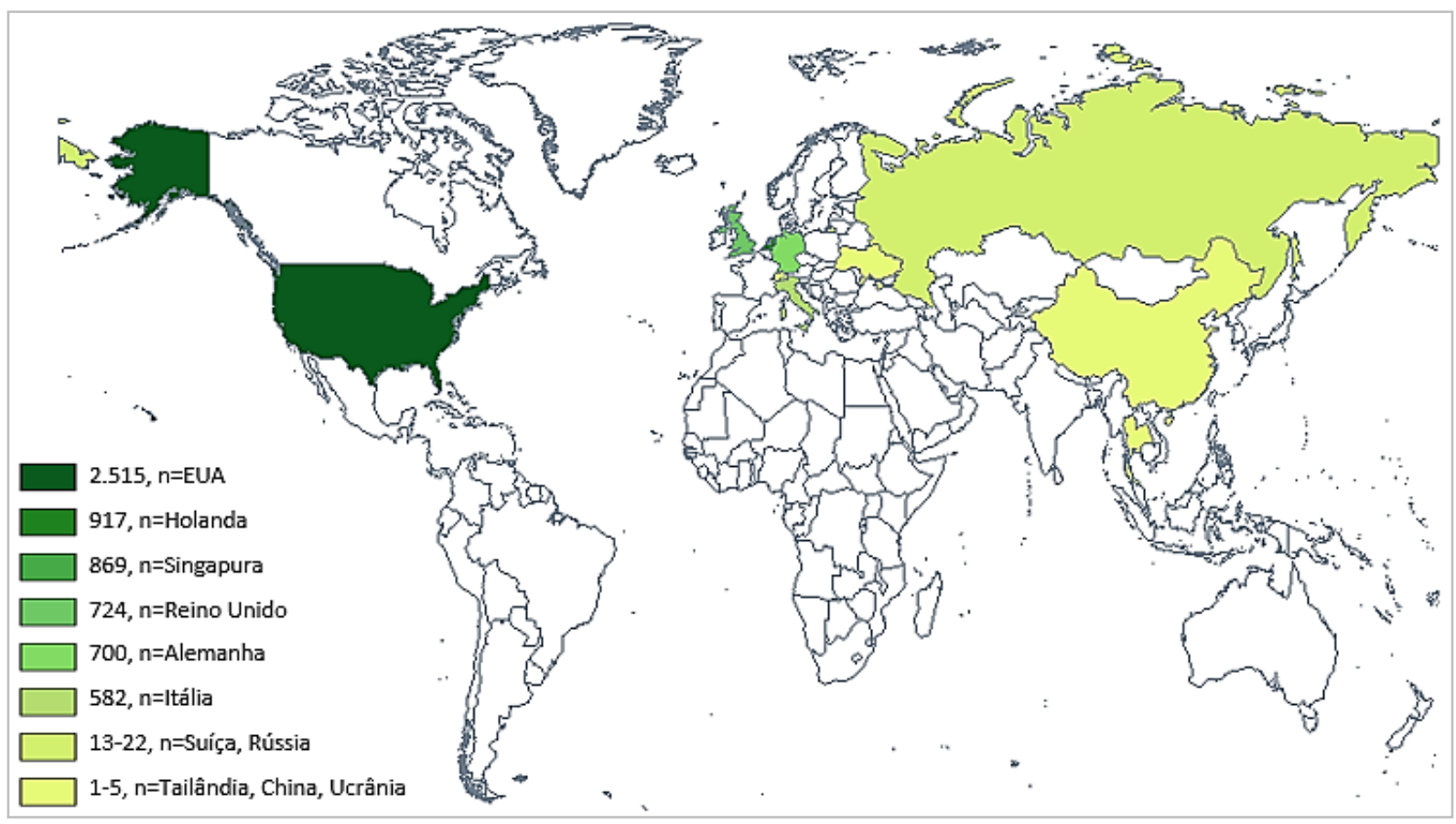

Figura 2. Distribuição geográfica da produção científica da FAE brasileira indexada na WoS conforme país editor dos artigos (1983-2013)

Fonte: dados da pesquisa

Os periódicos de maior tradição na FAE como Physical Review D, Journal of High Energy Physics e European Physical Journal C foram os preferidos pelos autores brasileiros para a publicação dos resultados científicos (Tabela 1). 70\% dos artigos foram publicados em seis títulos, denotando a alta concentração da pesquisa em poucas revistas especializadas. Outros autores como Mele et. al (2006), Calero (2009) e Aman (2013) já tinham reconhecido anteriormente essa particularidade da área.

Tabela 1. Principais periódicos de publicação dos artigos da FAE brasileira indexados na WoS (19832013)

\begin{tabular}{ccccccc}
\hline Periódico & N. artigos & $\%$ & $\mathbf{\Sigma} \%$ & País & Idioma & FI (2014) \\
\hline Phys. Rev. D & 2.259 & 35,57 & 35,57 & EUA & Inglês & 4.643 \\
J. High Energy Phys & 505 & 7,95 & 43,52 & Itália & Inglês & 6.111 \\
Eur. Phys. J. C & 449 & 7,07 & 50,59 & Alemanha & Inglês & 5.084 \\
Nucl. Instr. Meth. A & 435 & 6,85 & 57,44 & Holanda & Multilíngue & 1.216 \\
Int. J. Mod. Phys. A & 382 & 6,02 & 63,46 & Singapura & Inglês & 1.699 \\
Mod. Phys. Lett. A & 354 & 5,57 & 69,03 & Singapura & Inglês & 1.198 \\
Class. Quantum Grav. & 342 & 5,39 & 74,42 & Inglaterra & Multilíngue & 3.168 \\
\hline C) RDBCI: Rev. Digit. Bibliotecon. Cienc. Inf. & Campinas, SP & v.15 & n.1 & p. 37-52 & jan./abr. 2017 \\
\hline
\end{tabular}




\begin{tabular}{ccccccc}
\hline Nucl. Phys. B & 297 & 4,68 & 79,10 & Holanda & Inglês & 3.929 \\
J. Phys. G & 232 & 3,65 & 82,75 & Inglaterra & Inglês & 2.777 \\
Gen. Relat. Gravit. & 187 & 2,94 & 85,69 & EUA & Inglês & 1.771 \\
\hline
\end{tabular}

Fonte: WoS, Journal Citation Reports (JCR)

Os indicadores de difusão internacional confirmaram que os três periódicos mais produtivos no período são coincidentemente os que apresentam o fator de impacto mais alto, ubicando-se no quartil 1 da WC Physics, Particles \& Fields. No ranking de periódicos com maior FI dentro da categoria mencionada na WoS, os três títulos aparecem muito bem posicionados, ficando apenas atrás do Living Reviews in Relativity e do Annual Review of Nuclear and Particle Science. O patamar de excelência internacional alcançado pelos programas de pós-graduação brasileiros em FAE com nota máxima segundo a avaliação da Coordenação... (2013b) se manifesta com a publicação nas prestigiosas revistas da área. $\mathrm{Na}$ Figura 3 é possível observar a distribuição da produção científica por períodos segundo o periódico de publicação.

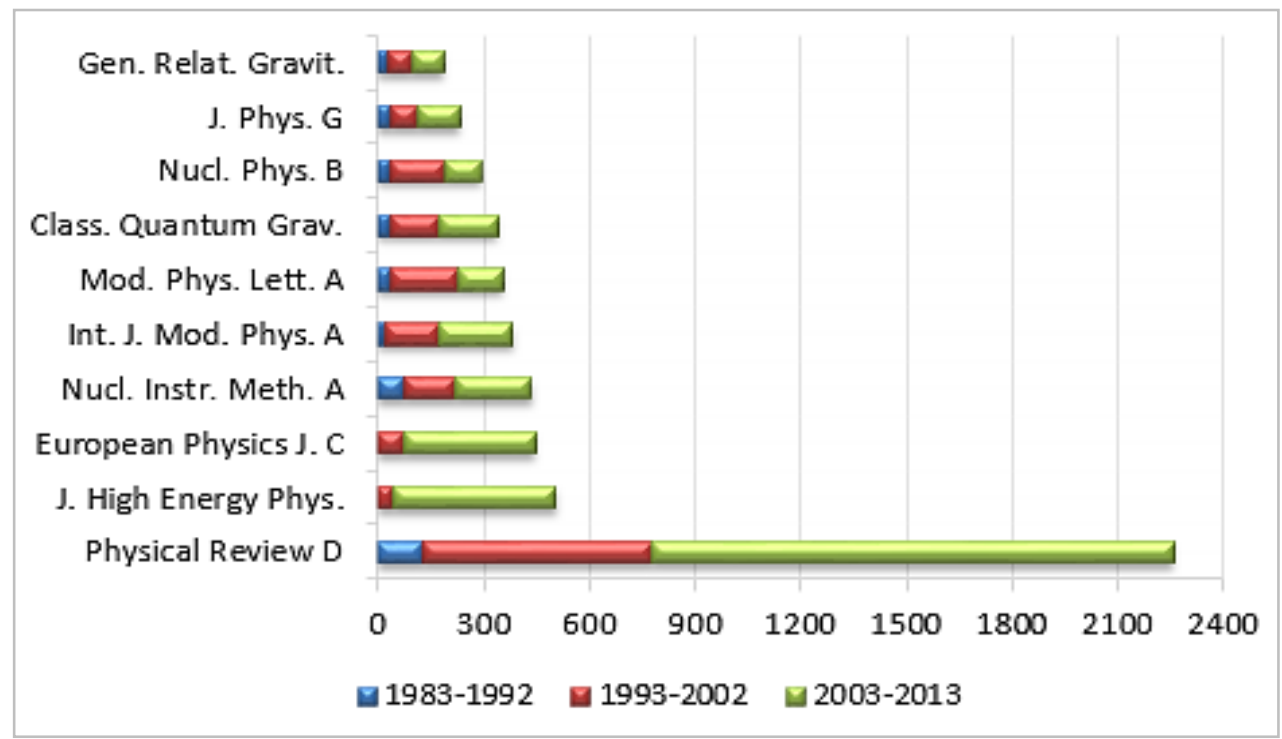

Figura 3. Distribuição dos artigos da FAE brasileira por períodos segundo o periódico de publicação (1983-2013)

Fonte: dados da pesquisa

Quando o conjunto de artigos da FAE foi agrupado por períodos, observou-se uma ligeira tendência ascendente do número de publicações por periódico do Q1 e Q2. Os resultados sugerem uma preferência inicial dos autores brasileiros pela publicação em revistas norte-americanas como é o caso da Physical Review D editado desde 1970 pela American Physical Society (APS). Ainda, é possível distinguir entre 1983 e 1992, outro veículo com alta popularidade dentro da comunidade científica, o Nuclear Instruments and Methods in Physics Research A. No período de 1993-2002, percebe-se o incipiente surgimento dos periódicos europeus Journal of High Energy Physics (SISSA/IOP) e European Physical Journal C (Springer). A consolidação desses canais de difusão das investigações brasileiras em FAE é claramente perceptível entre 2003 e 2013. 


\subsection{Colaboração Internacional}

Em seguida, a internacionalização da FAE foi analisada com base no percentual de artigos publicados conjuntamente entre o Brasil e outros países. O número de documentos em colaboração internacional da FAE brasileira no SCI entre 1983 e 2013 é de 2.888 artigos. Considerando a amplitude da coautoria, as publicações assinadas pelo Brasil e outro país (colaboração bilateral) alcança 1.371 artigos (47,47\%). Já as assinadas por três países (colaboração trilateral) 441 artigos $(15,27 \%)$ e as assinadas por $\geq 4$ países (colaboração multilateral) 1.076 artigos (37,26\%). Quando as publicações em colaboração internacional foram reagrupadas, observou-se que os artigos assinados entre 2 a 5 países representam $69,39 \%$ da totalidade. Já os artigos assinados por $\geq 6$ países representam 30,61\% das publicações, confirmando a presença do Brasil nas grandes redes de coautoria.

A proporcão de artigos da FAE assinados por mais de um país $(49,07 \%)$ é amplamente superior aos índices de colaboração internacional verificados na produção científica brasileira em anos recentes, onde a coautoria com autores de outros países ocorreu em aproximadamente 30\% das publicações (LETA; THIJS; GLÄNZEL, 2013). Em nível institucional, verificou-se que $31,74 \%$ das publicações foram assinadas por uma única instituição, enquanto que 19,19\% dos artigos estão vinculados à colaboração nacional. No decorrer do período, vislumbra-se um maior crescimento do número de publicações em colaboração internacional do que em colaboração nacional e sem colaboração, principalmente a partir de 2008, coincidindo com o ano de instituição da RENAFAE (Figura 4).

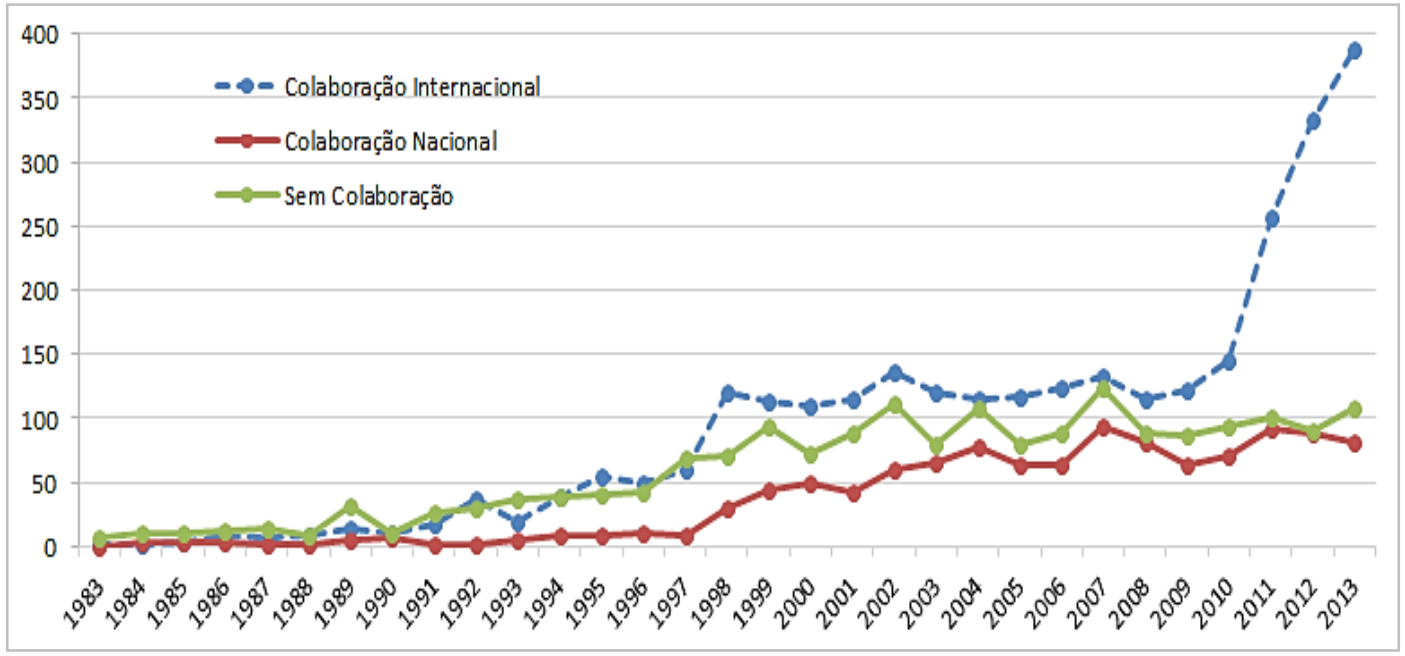

Figura 4. Dinâmica de crescimento da produção científica da FAE segundo o tipo de colaboração (1983-2013)

Fonte: dados da pesquisa

Para a viabilização dos experimentos relacionados com os aceleradores e detectores de partículas, a FAE requer a utilização conjunta de grandes instalações e instrumentos complexos, demandando a participação de especialistas de diferentes áreas e o aporte econômico de vários países (VANZ; STUMPF, 2010). De acordo com Leta e Cruz (2003), a

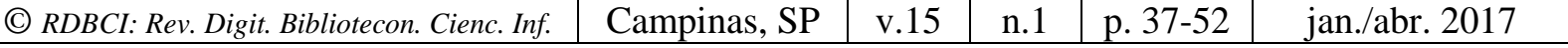


redução de custos nas pesquisas e a multidisciplinaridade de algumas áreas são provavelmente os aspectos que mais estimulam a colaboração científica. Braun et al. (1992) afirmam que nas Ciências Espaciais, FAE, Astrofísica e Física Nuclear o custo das investigações é superior ao orçamento local da maioria dos países. Os artigos publicados em colaboração internacional foram assinados por autores de 74 países, incluído o Brasil. A distribuição geográfica da produção científica da FAE indexada na WoS conforme país colaborador permite visualizar parceiros de diferentes continentes e inferir sobre a dimensão da internacionalização das publicações brasileiras (Figura 5).

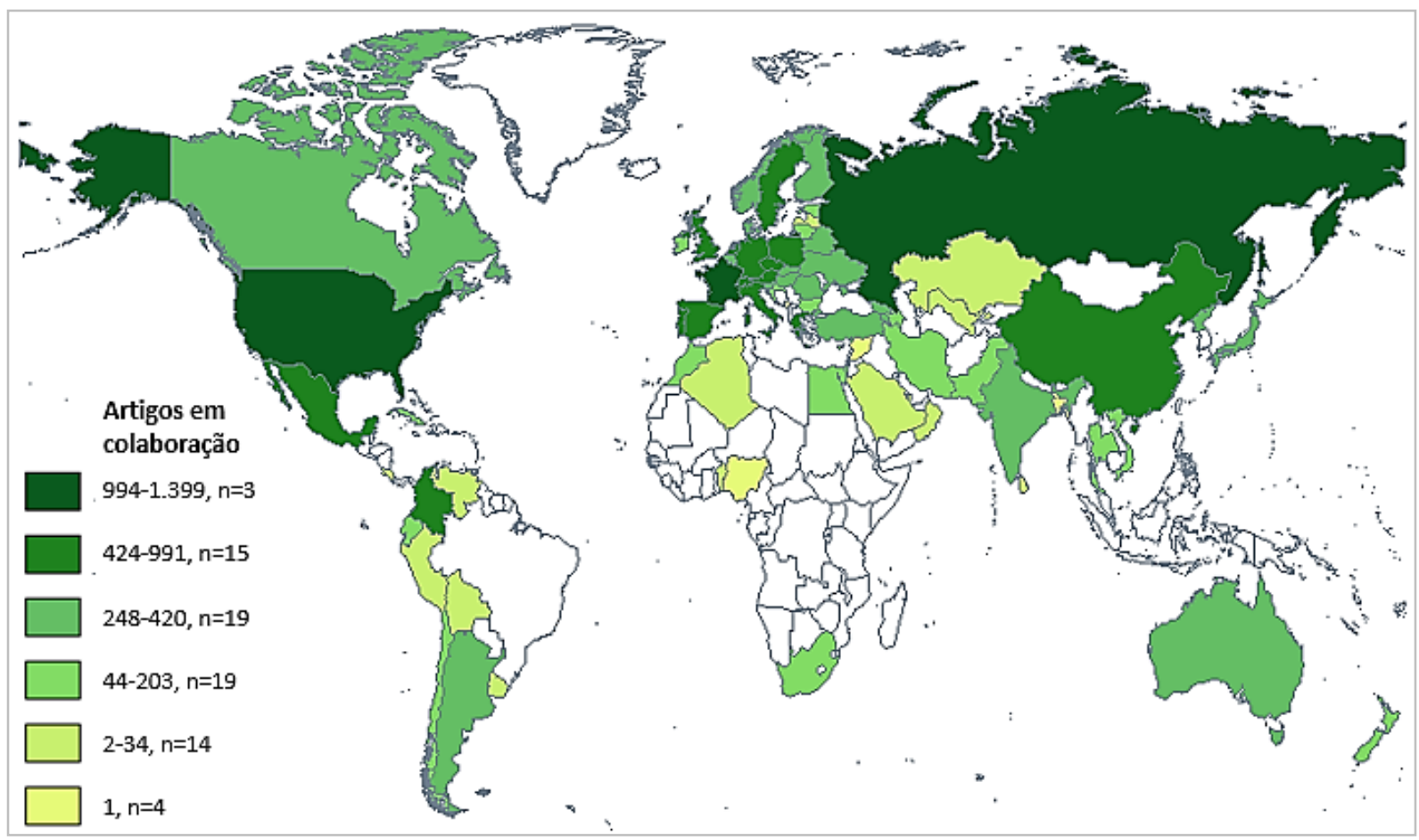

Figura 5. Distribuição geográfica da produção científica da FAE brasileira indexada na WoS conforme país colaborador (1983-2013)

Fonte: dados da pesquisa

Dentre os coautores do Brasil em FAE no período de 1983-2013, destacam-se aqueles com maior tradição na pesquisa da área, Estados Unidos (6,10\%), Rússia (4,56\%), França (4,34\%), Alemanha (4,32\%), Reino Unido (4,19\%) e Itália (4,18\%). A intensidade da colaboração do Brasil com parceiros líderes em termos de produção mundial da Física de Altas Energias é mais perceptível no período de 2003-2013 (Figura 6). Os principais países colaboradores da FAE são basicamente os mesmos que foram identificados por Vanz (2009) nas coautorias da ciência brasileira. O predomínio das relações de cooperação com os parceiros citados pode ser explicado pelos acordos e programas de apoio à pesquisa colaborativa internacional que são firmados pelo Brasil. Com os Estados Unidos, mantem-se convênios de colaboração desde 1950; com a Alemanha desde 1964; com a França, Portugal e Reino Unido desde 1968; com a Holanda desde 1971; com a China desde 1982; com a Espanha desde 1992; com a Itália desde 1998 (AGÊNCIA..., 2016, documento on-line). 


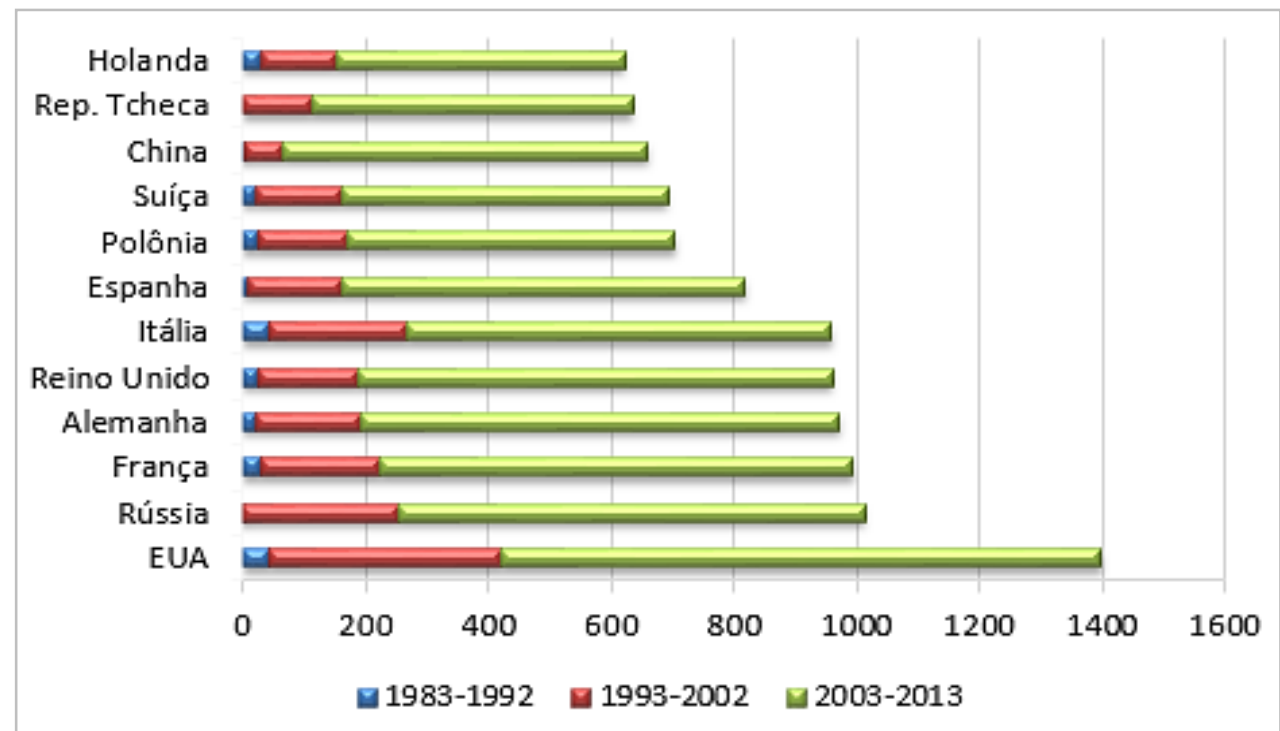

Figura 6. Distribuição das publicações da FAE brasileira por períodos segundo o país colaborador (1983-2013)

Fonte: dados da pesquisa

A baixa participação dos Estados Unidos percebida nos artigos da FAE implica uma redução da dependência científica do Brasil com esse país e uma consolidação dos vínculos com parceiros, na sua maioria, da Europa. Esse indicador ratifica a tendência nacional (todas as áreas) observada no estudo de Vanz (2009). Os grandes projetos internacionais outorgam à comunidade científica brasileira a oportunidade, principalmente em laboratórios americanos e europeus, de novas investigações em colaboração com diversos países no campo da Física de Partículas.

\subsection{Impacto Internacional}

Por último, a internacionalização da FAE brasileira foi estimada a partir do percentual de documentos citantes estrangeiros. Os 6.350 artigos da produção científica receberam 78.812 citações provenientes de 41.152 documentos, com média de crescimento de 32,17\% trabalhos ao ano. Em termos de impacto, infere-se que a forte internacionalização da pesquisa na área contribuiu para a elevada porcentagem de artigos citados (87,65\%). Lehmann, Laudrup e Jackson (2003) afirmam que devido ao custo operacional dos experimentos, ao controle rigoroso da pesquisa e ao grande número de coautores é de se esperar uma menor fração de artigos minimamente citados em FAE.

As características gerais dos documentos citantes revelam a forte prevalência do artigo de periódico $(80,48 \%)$ dentre os tipos de documentos e do inglês $(99,70 \%)$ dentre os idiomas de publicação. A revista científica foi o canal de difusão mais utilizado pelos pesquisadores citantes. Em análise do conjunto de 35 títulos que publicaram 165 ou mais artigos, observou-se que $97,14 \%$ dos documentos publicados estão concentrados em veículos internacionais, com destaque para os periódicos editados na Europa $(49,80 \%)$ e nos Estados Unidos $(41,45 \%)$. Apenas uma revista brasileira foi identificada entre as mais produtivas, a 
Brazilian Journal of Physics. Physical Review D, Journal of High Energy Physics, Nuclear Physics B, Physics Letters B, European Physical Journal C e Physical Review Letters publicaram conjuntamente $44,06 \%$ dos documentos.

Da totalidade de documentos citantes, $39.555(96,11 \%)$ apresentaram dados sobre a filiação do autor. Quanto à procedência, constatou-se que $34.721(87,78 \%)$ pertencem aos citantes internacionais, sendo 30.327 (76,67\%) sem autores brasileiros e $4.394(11,11 \%)$ com autores brasileiros. Igualmente, os citantes nacionais respondem por 4.834 documentos $(12,22 \%)$ entre 1983 e 2014. A superioridade de pesquisadores filiados a instituições estrangeiras nos documentos citantes ressalta a internacionalização da pesquisa e a baixa autocitação em FAE. Os documentos citantes internacionais (sem autores brasileiros) apresentaram um crescimento visivelmente superior ao exibido pelos documentos citantes internacionais (com autores brasileiros) e documentos citantes nacionais durante o período (Figura 7).

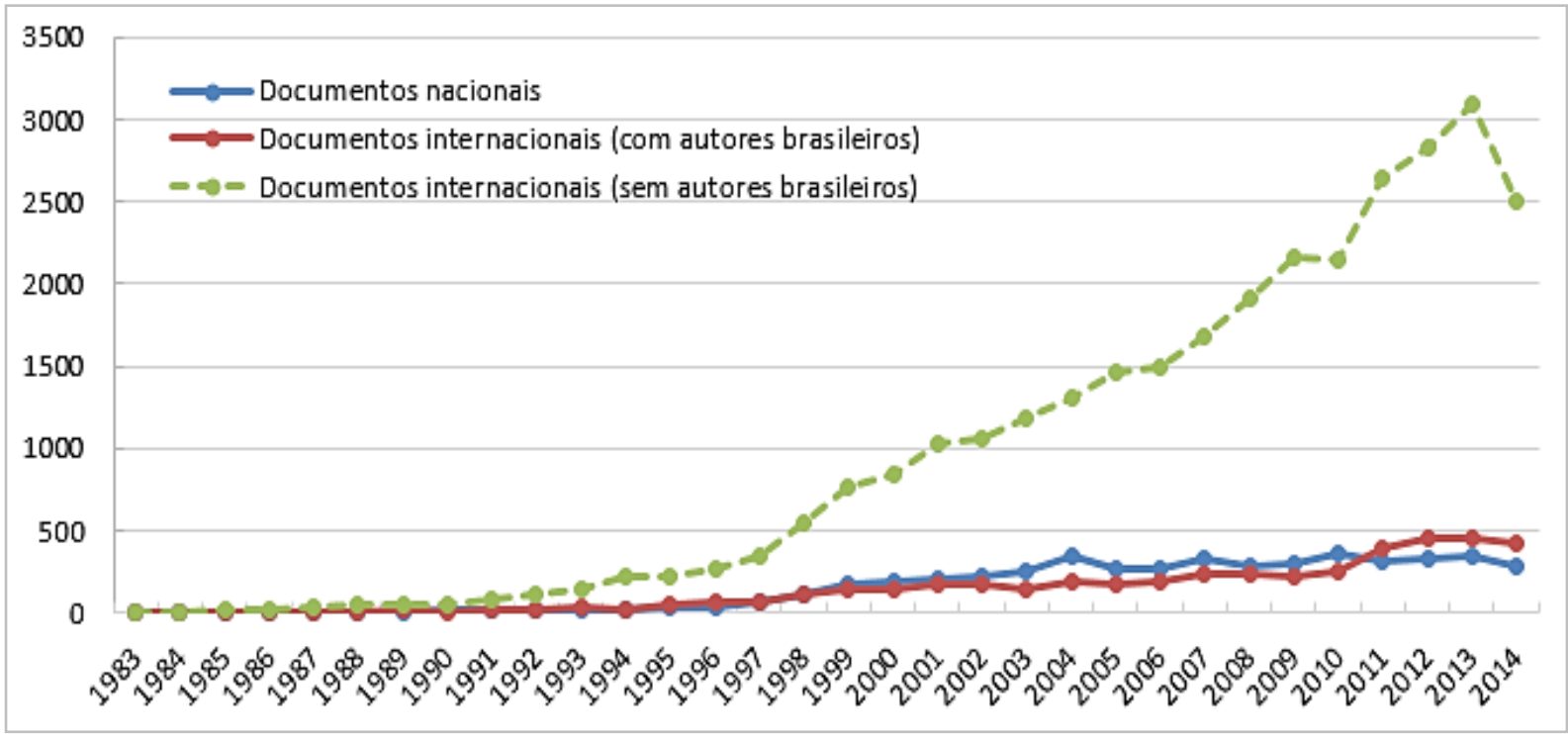

Figura 7. Dinâmica de crescimento dos documentos citantes da produção científica da FAE segundo a procedência (1983-2013)

Fonte: dados da pesquisa

Os cinco países citantes da produção científica da FAE brasileira com maior volume de publicações são Estados Unidos com 10.554 documentos (10,25\%), Brasil com 9.309 (9,04\%), Alemanha com 6.322 (6,14\%), Itália com 5.139 (4,99\%) e Reino Unido com 4.581 $(4,45 \%)$. A quantidade de citações desses países pode estar associada à posição de privilégio que eles ocupam na rede de colaboração internacional da Física de Altas Energias e no ranking mundial de produtividade. $\mathrm{O}$ estudo mostra claramente como o impacto da pesquisa do Brasil em FAE, medido através do número de citações por documento, é mais saliente entre 2004 e 2014, coincidindo com o período da criação da RENAFAE e do aumento da participação brasileira nas grandes colaborações internacionais (Figura 8). 


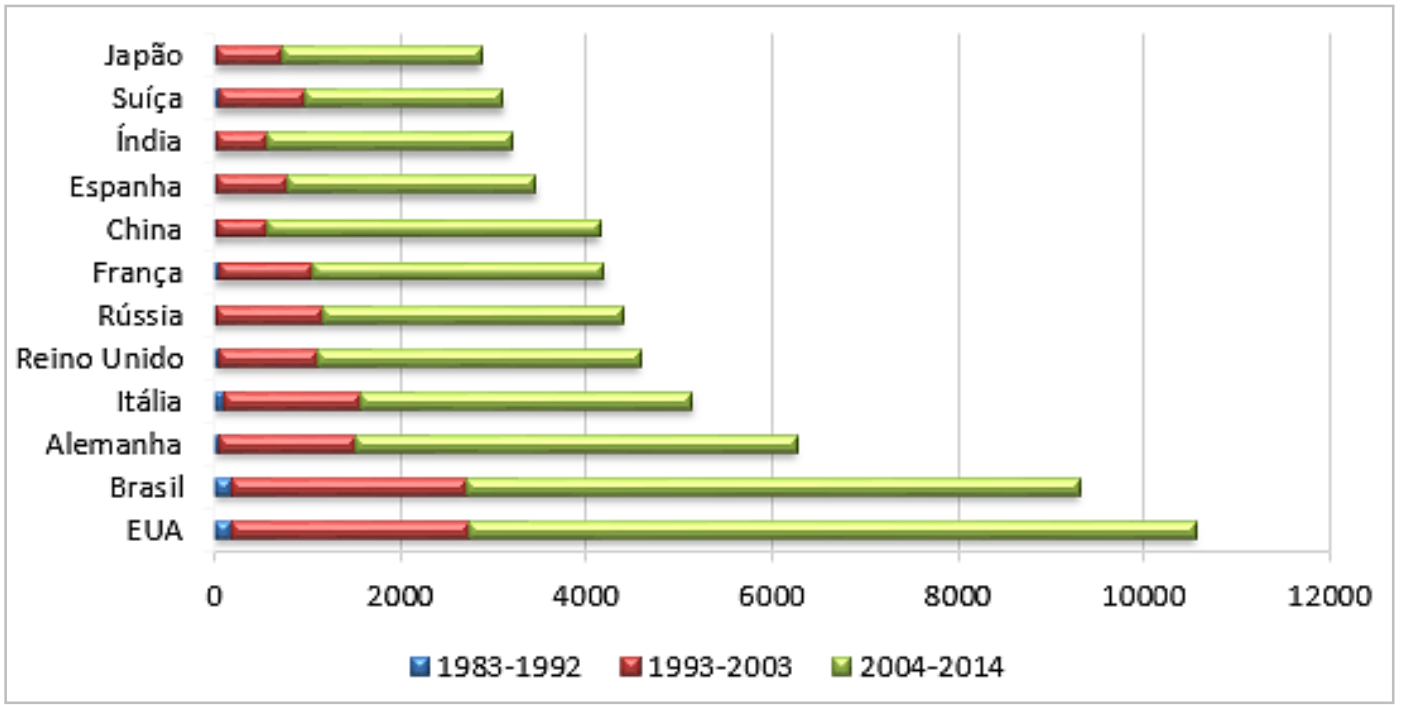

Figura 8. Distribuição das citações da produção científica da FAE por períodos segundo o país citante (1983-2013)

Fonte: dados da pesquisa

A produção da FAE brasileira foi citada por 112 países (incluído o Brasil), revelando a influência e o prestígio dos autores brasileiros dentro da comunidade científica internacional. A Figura 9 exibe a distribuição geográfica dos documentos citantes e a intensidade do impacto da atividade científica do Brasil em FAE no mundo.

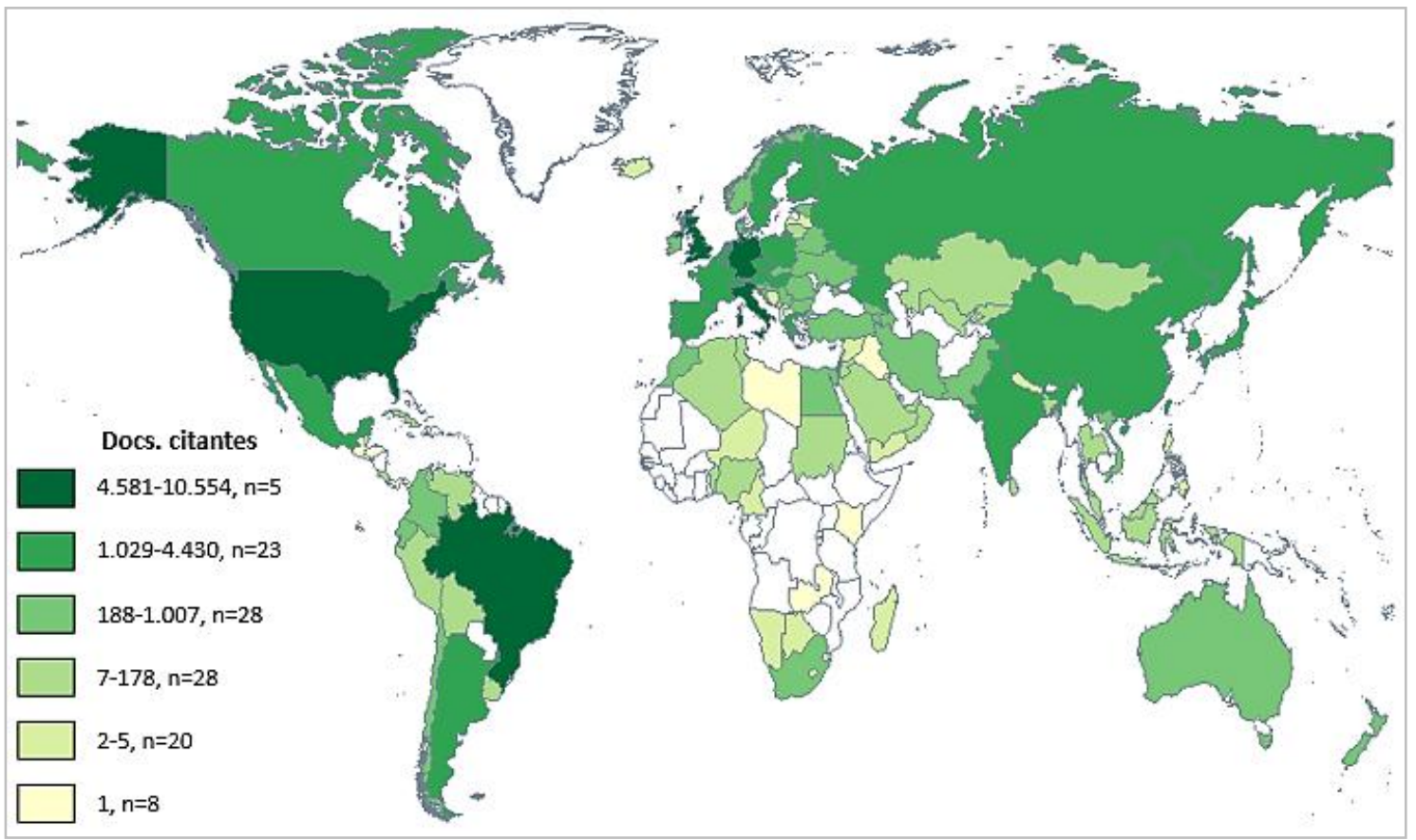

Figura 9. Distribuição geográfica dos documentos citantes da produção científica da FAE brasileira conforme país de origem (1983-2014)

Fonte: dados da pesquisa

O exame do alcance da pesquisa brasileira indexada na WoS também foi examinada em relação aos continentes dos documentos citantes. A Europa, principal coautora nos artigos da FAE no período 1983-2013, é também a região que concentra o maior número de citações, 55.579 (53,98\%). A supremacia de citações do continente europeu está atrelada à

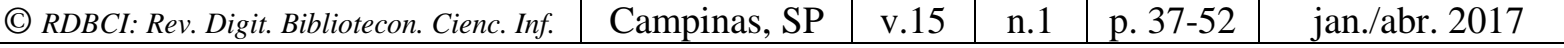


contribuição científica dos Estados-Membros do CERN e à maior tradição na pesquisa experimental de alguns países como Alemanha e Itália. A continuação, ubicaram-se América com 27.259 citações (26,47\%), Ásia com 17.604 (17,09\%), África com 1.421 (1,38\%) e Oceania com $1.117(1,08 \%)$.

\section{CONSIDERAÇÕES FINAIS}

Nesta pesquisa foi possível inferir que o aumento da produção científica da FAE brasileira indexada na WoS no período de 1983-2013 pode ter sido decorrência da expansão da pesquisa a partir da ampliação do número de programas de pós-graduação em Astronomia e Física com linhas de pesquisa $\mathrm{FAE}$, grupos $\mathrm{CNPq}$, intensificação das colaborações internacionais do Brasil e instituição da RENAFAE. Com relação à análise da dimensão da internacionalização da atividade científica em FAE através de indicadores de difusão, colaboração e impacto internacional, os resultados mostram uma crescente presença dos pesquisadores brasileiros nas principais revistas internacionais de alto fator de impacto da Física de Altas Energias, atendendo ao modelo de internacionalização e desenvolvimento científico para países proposto por van Raan (1997). A proporção de artigos da FAE escritos por mais de um país é amplamente superior aos índices de colaboração internacional verificados na produção científica brasileira nos últimos anos, confirmando a presença do Brasil nas grandes redes multinacionais. As análises mostram um nótorio crescimento dos documentos citantes internacionais e um baixo percentual de autocitação no período. Tanto a distribuição do número de coautorias e citações internacionais da FAE sugerem uma forte correlação entre a colaboração e o impacto internacional. Os indicadores de internacionalização utilizados nesta pesquisa desvelaram o perfil científico internacional da FAE no Brasil, acompanhando o padrão característico da disciplina na esfera global. Os resultados alcançados contribuem para o conhecimento das dimensões internacionais da FAE brasileira e fornecem subsídios para o planejamento e aperfeiçoamento de políticas e estratégias vinculadas à negociação para o ingresso do Brasil no CERN sob uma nova modalidade de participação, a ampliação dos investimentos, a criação de infraestrutura nacional, a ampliação da colaboração internacional, a expansão dos programas de pósgraduação e atividades dos grupos de pesquisa $\mathrm{CNPq}$. 


\section{INTERNACIONALIZACIÓN DE LA PRODUCCIÓN CIENTÍFICA DE BRASIL EN FíSICA DE ALTAS ENERGÍAS (1983-2013)}

RESUMEN: Este estudio bibliométrico analiza las dimensiones de la internacionalización de la producción científica brasileña en Física de Altas Energías (FAE) a partir de artículos publicados en revistas indexadas por la Web of Science (WoS) en el período de 1983-2013. Con base en la clasificación propuesta por el Manual de Santiago de la Red de Indicadores de Ciencia y Tecnologia Iberoamericana e Interamericana (RICYT), la internacionalización en FAE es estimada a través de Indicadores de Difusión, Colaboración e Impacto Internacional. Los indicadores de Difusión Internacional muestran la preferencia por la publicación en revistas extranjeras de lengua inglesa. La totalidad de los artículos analizados fue publicada en 35 revistas de 11 países diferentes. Los indicadores de Colaboración Internacional muestran la prevalencia de la co-autoría en FAE dado que 49,07\% de los artículos fueron firmados por más de un país. Los indicadores de Impacto Internacional destacan el peso de las publicaciones en periódicos extranjeros de lengua inglesa entre los citantes. Los documentos citantes internacionales representan $87,78 \%$ del total, destacándose los firmados por autores de los Estados Unidos. Los indicadores de internacionalización utilizados desvelan el perfil científico internacional de la FAE en el Brasil, acompañando el modelo característico del área en la esfera global.

PALABRAS CLAVE: Producción científica. Internacionalización. Bibliometría. Física de Altas Energías.

Submetido em: 02-08-2016

Aceito em: 12-12-2016

Publicado em: 15/12/2016

\section{REFERÊNCIAS}

AGÊNCIA BRASILEIRA DE COOPERAÇÃO. Acordos vigentes. Brasília, DF: ABC, 2015. Disponível em: 〈http://www.abc.gov.br/CooperacaoTecnica/AcordosVigentes>. Acesso em: 13 jul. 2015.

AMAN, V. The potential of preprints to accelerate scholarly communication: a bibliometric analysis based on selected journals. arXiv preprint arXiv:1306.4856, 2013. Disponível em: <http://arxiv.org/abs/1306.4856>. Acesso em: 16 set. 2015.

BRAUN, T. et al. International co-authorship patterns in physics and its subfields, 19811985. Scientometrics, Amsterdam, v. 24. n. 2, 181-200, 1992. 
CALERO, A. I. B. La colaboración e la visibilidad en las disciplinas de Física en Science Citation Index y arXiv (2000-2005). 2009. 476 f. Tese (Doutorado) - Universidad Carlos III de Madrid, Departamento de Biblioteconomía y Documentación, Getafe, 2009.

COORDENAÇÃO DE APERFEIÇOAMENTO DE PESSOAL DE NÍVEL SUPERIOR.

Documento de área 2013. Brasília, DF: CAPES, 2013a. Disponível em: <http://www.capes. gov.br/images/stories/download/avaliacaotrienal/Docs_de_area/Astronomia_Fisica_ATT27S ET.pdf>. Acesso em: 29 jun. 2015.

COORDENAÇÃO DE APERFEIÇOAMENTO DE PESSOAL DE NÍVEL SUPERIOR. Relatório de avaliação 2010-2012 Trienal 2013. Brasília, DF: CAPES, 2013b. Disponível em: <http://www.capes.gov.br/component/content/article/44-avaliacao/4652astronomiafisica $>$. Acesso em: 24 ago. 2015.

CENTRO BRASILEIRO DE PESQUISAS FÍSICAS. Plano Diretor do CBPF 2011-2015. Rio de Janeiro: CBPF, 2011. Disponível em: <http://portal.cbpf.br/attachments/o_cbpf /pdfs/PlanoDiretor.pdf>. Acesso em: 23 nov. 2015.

CHAVES, A. et al. Física para um Brasil Competitivo. Brasília, DF: SBF, 2007.

DUARTE, R. P. Cooperação Internacional para o Desenvolvimento em Ciência e Tecnologia: a participação brasileira na Organização Europeia para Pesquisa Nuclear (CERN). Journal of Technology Management \& Innovation, v. 3, n. 4, p. 133-151, 2008.

KRAUSE, J.; LINDQVIST, C. M.; MELE, S. Quantitative study of the geographical distribution of the authorship of High-Energy Physics journals. CERN-OPEN-2007-014, 2007. Disponível em: <http://cds.cern.ch/record/1033099/files/cer002691702.pdf?origin =publication_detail>. Acesso em: 22 dez. 2015.

KRETSCHMER, H.; ROUSSEAU, R. Author inflation leads to a breakdown of Lotka's law. Journal of the American Society for Information Science and Technology, New York, v. 52, n. 8, p. 610-614, 2001.

LEHMANN, S.; LAUTRUP, B.; JACKSON, A. D. Citation Networks in High Energy Physics. Physical Review E, v. 68, n. 2, 2003.

LEITE, P.; MUGNAINI, R.; LETA, J. A new indicator for international visibility: exploring Brazilian scientific community. Scientometrics, Amsterdam, v. 88, n. 1, p. 311-319, 2011.

LETA, J.; CRUZ, C. H. de B. A produção científica brasileira. In: VIOTTI, E. B.; MACEDO, M. M. Indicadores de Ciência, Tecnologia e Inovação no Brasil. Campinas: Ed. Unicamp. 2003. p. 121-168.

LETA, J.; THIJS, B.; GLÄNZEL, W. A macro-level study of science in Brazil: seven years later. Encontros Bibli, Florianópolis, v. 18, n. 36, p. 51-66, jan./abr. 2013.

MELE, S. et al. Quantitative analysis of the publishing landscape in High-Energy Physics. Journal of High Energy Physics, v. 12, p. 1-25, 2006. 
RED DE INDICADORES DE CIENCIA Y TECNOLOGÍA IBEROAMERICANA E INTERAMERICANA. Manual de indicadores de internacionalización de la ciencia y la tecnología. Buenos Aires: RICYT, 2007. Disponível em: <http://www.oei.es/salactsi/manual _santiago.pdf>. Acesso em: 16 maio 2016.

SANTIN, D. M.; VANZ, S. A. de S; STUMPF, I. R. C. Internacionalização da produção científica em Ciências Biológicas da UFRGS: 2000-2011. TransInformação, Campinas, v. 27, n. 3, p. 209-218, set./dez., 2015.

SHELLARD, R. C. Física de Altas Energias no Brasil. Tlaxcala: CBPF, 2011.

VAN RAAN, A. F. J. Science as an international enterprise. Science and Public Policy, v. 24, n. 5, p. 290-300, 1997.

VANZ, S. A. de S. As redes de colaboração científica no Brasil: (2004-2006). 2009. 204 f. Tese (Doutorado)-Universidade Federal do Rio Grande do Sul, Faculdade de Biblioteconomia e Comunicação, Porto Alegre, 2009.

VANZ, S. A. de S; STUMPF, I. R. C. Colaboração científica: revisão teórico conceitual. Perspectivas em Ciência da Informação, Belo Horizonte, v. 15, n. 2, p. 42-55, maio./ago. 2010.

WANG, S.; WANG, H.; WELDON, P. R. Bibliometric analysis of English-language academic journals of China and their internationalization. Scientometrics, v. 73, n. 3, p. 331343, 2007.

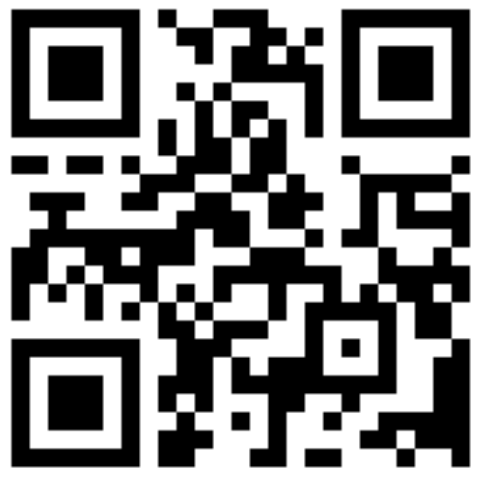

